\title{
Les trames vertes urbaines, un nouveau support pour une cité verte?
}

Urban green infrastructures : a new basis for a green city?

Jean-Michel Le Bot et Françoise Philip

\section{(2) OpenEdition}

\section{Journals}

Édition électronique

URL : http://journals.openedition.org/developpementdurable/9318

DOI : 10.4000/developpementdurable.9318

ISSN : 1772-9971

Éditeur

Association DD\&T

Référence électronique

Jean-Michel Le Bot et Françoise Philip, « Les trames vertes urbaines, un nouveau support pour une cité verte? », Développement durable et territoires [En ligne], Vol. 3, n² 2 | Juillet 2012, mis en ligne le 12 juillet 2012, consulté le 20 avril 2019. URL : http://journals.openedition.org/ developpementdurable/9318; DOI : 10.4000/developpementdurable.9318

Ce document a été généré automatiquement le 20 avril 2019

Développement Durable et Territoires est mis à disposition selon les termes de la licence Creative Commons Attribution - Pas d'Utilisation Commerciale 4.0 International. 


\section{Les trames vertes urbaines, un nouveau support pour une cité verte?}

Urban green infrastructures : a new basis for a green city?

Jean-Michel Le Bot et Françoise Philip

Au début des années 1990, C. Lafaye et $\mathrm{L}$. Thévenot (1993) se demandaient s'il n'était pas possible de définir un septième mode de justification, celui de la cité verte, aux côtés des six autres définis par L. Boltanski et L. Thévenot $(1991)^{1}$. Ils relevaient en effet que «les préoccupations en matière d'environnement constitu[ai]ent une ressource active pour questionner et mettre en cause, sur un certain nombre de points précis, la légitimité des autres modes d'évaluation et de justification à dire le bien commun » (Lafaye et Thévenot, 1993 : 512). Leur conclusion, toutefois, était que "la grandeur verte» paraissait «encore insuffisamment outillée pour servir largement dans des justifications ordinaires et permettre leur mise à l'épreuve » (ibid. : 513). Ils soulignaient par ailleurs la radicale nouveauté introduite par la question écologique par rapport aux régimes antérieurs de justification. En effet, les débats et conflits écologiques ne s'organisaient déjà plus seulement autour de la défense de personnes ou de collectifs humains. Ils débordaient l'humanité pour inclure la défense d'êtres non humains. La césure traditionnelle entre l'ordre de la culture et l'ordre de la nature se voyait donc remise en cause (Latour, 1991; Descola, 2005). La question écologique pouvait ainsi déboucher sur un élargissement du collectif, incluant désormais des non-humains (dont des espèces végétales et animales). Mais cet élargissement reposait souvent sur des notions issues de la biologie comme la notion d'écosystème, avec, au plus haut niveau, l'écosystème global constitué par la biosphère, le risque étant alors de donner dans les débats un poids particulier au savoir des experts, seuls à même de s'élever à ce niveau surplombant du système. Cela conduisait les auteurs à ne pas aller au-delà de l'hypothèse d'une "nouvelle cité en cours de construction " (Lafaye et Thévenot, 1993 : 510). 
Qu'en est-il près de vingt ans plus tard? Les réflexions et débats autour de la mise en œuvre de trames vertes urbaines nous ont paru propices à réexaminer cette question. La trame verte et bleue en tant que projet national issu du Grenelle de l'environnement est en effet devenue un outil de l'aménagement du territoire ${ }^{2}$. Elle a " pour objectif d'enrayer la perte de biodiversité en participant à la préservation, à la gestion et à la remise en bon état des milieux nécessaires aux continuités écologiques, tout en prenant en compte les activités humaines, et notamment agricoles, en milieu rural ». Pour cela, ces trames doivent contribuer en premier lieu à « diminuer la fragmentation et la vulnérabilité des habitats naturels et habitats d'espèces et prendre en compte leur déplacement dans le contexte du changement climatique " ainsi qu'à «identifier, préserver et relier les espaces importants pour la préservation de la biodiversité par des corridors écologiques » ${ }^{4}$. Les notions de "préservation de la biodiversité » et de "continuité écologique» se trouvent donc inscrites dans le droit. Le mode de légitimation écologique dont Lafaye et Thévenot soulignaient en 1993 la radicale nouveauté semble ainsi avoir gagné en puissance. A-t-il conquis son autonomie par rapport aux cités déjà constituées?

Pour contribuer à répondre à cette question, cet article s'appuie sur une enquête menée dans trois agglomérations françaises (Angers, Nantes et Rennes) dans le cadre de la tâche $3 \mathrm{du}$ programme de recherche Trame Verte Urbaine ${ }^{5}$. Si ces agglomérations ne sont pas nécessairement représentatives de l'ensemble des démarches mises en place sur le territoire national, elles permettent néanmoins de donner un aperçu de la diversité des actions menées pour la mise en œuvre des trames vertes, dont on sait qu'elles mobilisent des intérêts hétérogènes et souvent divergents (Berges et al., 2010). L'enquête a consisté en entretiens semi-directifs réalisés auprès de représentants institutionnels et d'acteurs locaux intervenant directement dans les projets de trames vertes de ces agglomérations : directeurs de services d'urbanisme, d'aménagement du territoire ou de services des jardins, chargés de mission, urbanistes, élus, responsables d'associations de protection de l'environnement (26 personnes interrogées).

4 L'article s'appuiera sur l'analyse de ces entretiens pour examiner la façon dont les argumentations au sujet de la trame verte viennent s'inscrire dans les six ordres de justification préexistants : celui du monde industriel, du monde civique, du monde de l'opinion, du monde domestique, du monde marchand et du monde inspiré (Boltanski et Thévenot, 1991). L'ordre de présentation retenu est différent de celui qu'avaient adopté Boltanski et Thévenot (1991), ainsi d'ailleurs que Godard (1990) ou Lafaye et Thévenot (1993). Il tient compte en effet de la plus ou moins grande présence des différents ordres de justification dans les entretiens.

\section{Le monde industriel}

5 En tant que « ressource naturelle à exploiter » (Godard, $1990: 224)$, la nature a toute sa place dans le monde industriel. Elle y est «grande» dans la mesure où "[son] énergie et [sa] puissance se trouvent maîtrisées, [où elle] est rendue prévisible, utile et fonctionnelle, [où elle] répond à des besoins, [où elle constitue] une "nature" objective, appréhendée par des scientifiques et des ingénieurs en vue de son usage » (ibid.). Or la critique écologiste s'est élevée contre cette idée d'une nature à exploiter. Elle a contribué à mettre en avant l'idée d'une nature qu'il faut défendre pour elle-même, indépendamment donc de toute finalité industrielle. De fait, pour les associations naturalistes rencontrées, la trame verte pourrait dans l'idéal intégrer un certain nombre d'espaces de nature dont l'accès serait interdit aux humains 
ou en tout cas fortement contrôlé et limité. Alors que les conceptions traditionnelles de la nature urbaine distinguent généralement une nature "civilisée " (la nature cultivée et jardinée des parcs et jardins) et une nature «sauvage " (les plantes spontanées et les friches qui, même mieux acceptées, restent contrôlées - Menozzi, 2007), certains défenseurs de la trame considèrent que la présence en ville de la nature spontanée se justifie pour elle-même en tant que réserve de biodiversité "ordinaire ", sans qu'il soit nécessaire de lui trouver une fonctionnalité sociale ou économique. À Angers par exemple, lors des ateliers thématiques organisés pour débattre de la première version du document d'orientation générale (DOG) du Schéma de cohérence territoriale (SCOT), les associations naturalistes ont souhaité que les «liaisons douces » (circuits de randonnées et cyclistes), que le DOG proposait d'améliorer et de développer, soient intégrées à la trame verte et bleue «afin que la sensibilité des milieux prévale à l'opportunité de création de telles installations ». Elles ont estimé que la vocation de la Loire, en particulier, était d'abord écologique et que "son accessibilité touristique [devait] être limitée et conditionnée par la sensibilité des milieux $»^{6}$.

6 Pour autant, Lafaye et Thévenot remarquaient déjà le développement d'une "tendance visant à internaliser les préoccupations écologiques au sein de l'argumentation technique " (Lafaye et Thévenot, 1993 : 509). « Les questions d'environnement » étaient alors « assimilées à des contraintes dont la réalisation de la structure ou de l'aménagement doit tenir compte en vue d'un "développement durable" " (ibid.). De même, Bruno Latour pouvait écrire que "les luttes contre les gaspillages [et] les nuisances", ainsi que le bouclage des cycles de production, pouvaient se ranger "sans difficulté dans la cité industrielle» par le biais de nouveaux appareillages et de nouvelles réglementations (Latour, 1995 : 10). Or c'est bien en termes d'internalisation des questions écologiques dans le monde industriel que l'on peut comprendre le compromis recherché par Angers Loire Métropole au sujet des liaisons douces : «Il faut trouver le niveau d'aménagement qui soit compatible avec la biodiversité parce que c'est pour ça qu'on fait la trame. Il n'est donc pas question de bitumer, de faire des trucs énormes... Il faut faire des aménagements qui soient adaptés aussi à cette vocation biodiversité, mais dans notre esprit, on ne ferme pas au public. Cette trame peut être aussi la base d'un réseau intéressant pour modes de déplacements doux. Ballade à pieds, la découverte du territoire, tout ça aussi peut être dans cette vocation trame verte " (Directrice adjointe à la direction du Développement des Territoires, Angers Loire Métropole). Un tel compromis engage sans doute le monde industriel avec le monde civique. Mais ce qu'il faut d'abord souligner, c'est qu'avec les trames vertes et les nouvelles politiques de préservation de la biodiversité, c'est la cité industrielle elle-même qui poursuit sa mutation. Certes, des pratiques comme la gestion différenciée, qui n'ont pas attendu la réflexion sur les trames vertes mais qui viennent s'y intégrer, impliquent une remise en cause de techniques et d'habitudes profondément ancrées chez les professionnels des espaces verts, techniques qui ont longtemps été considérées comme des symboles de "modernité " et de " progrès » telles que l'utilisation de pesticides de synthèse. Pourtant, il serait faux d'en conclure que ces nouvelles pratiques ne puissent pas trouver leur justification dans les termes du monde industriel. C'est plutôt ce dernier qui intègre désormais le génie écologique. L'ancien monde industriel valorisait les " produits phytosanitaires ${ }^{7}$ comme outils permettant de contrôler efficacement la nature, d'améliorer sa productivité et de maintenir une esthétique horticole agréable aux usagers. Dans le cadre du nouveau monde industriel, les trames vertes, destinées à préserver l'ensemble de la biodiversité, y compris donc la biodiversité "ordinaire ${ }^{8}$, et pas seulement quelques espèces 
emblématiques ou "patrimoniales », doivent être associées à des pratiques culturelles et culturales plus écologiques. Du coup, la préservation de la biodiversité et d'éléments de la trame est intégrée par la maîtrise d'ouvrage comme par la maitrise d'œuvre parmi ces « contraintes " dont il faut "tenir compte en vue d'un "développement durable" " (Lafaye et Thévenot, op. cit.). Cela apparaît très clairement dans différents projets urbains en cours sur les trois métropoles de l'enquête (particulièrement sur la ZAC de la Courrouze et la ZAC Maurepas-Gayeulles à Rennes).

7 Beaucoup de débats actuels nous semblent donc s'inscrire dans ce nouveau monde industriel. C'est le cas des réflexions qui déplorent que les documents d'urbanisme permettent «d'identifier les zones à protéger » mais restent muets «quand aux modalités de gestion à envisager sur les espaces concernés » (Cormier et al., 2010 : § 58). « La loi nous dit qu’il faut privilégier les outils [de] nature contractuelle pour mettre en cuvre la trame. Mais les outils de nature contractuelle en espace agricole, ce sont des mesures environnementales, elles sont ciblées dans les zones Natura 2000 et dans les zones prioritaires par rapport à l'eau. Quand on n'est pas dans ces zones-là, on n'a rien. Du coup, la biodiversité ordinaire en espace agricole, il n'y a pas vraiment de levier aujourd'hui. [...] Le problème c'est que l'entrée par les documents d'urbanisme ne parle justement pas de gestion. Elle parle de destination des sols, mais elle n'intervient en aucun cas sur la gestion des espaces en question. " (Chargé de mission trame verte et bleue à la Direction régionale de l'environnement, de l'aménagement et du logement - DREAL - des Pays-de-Loire). C'est le cas aussi de beaucoup de questions en amont sur les types d'habitats (au sens écologique) qui peuvent venir composer les trames. Les grandes cultures, par exemple, ne sont pas considérées comme une matrice de qualité pour les corridors écologiques (les associations naturalistes rencontrées le soulignent avec insistance). En raison de la monoculture, des traitements chimiques, de l'absence ou de la destruction du bocage, ces grandes cultures constitueraient en effet une véritable barrière à la dispersion des espèces ${ }^{9}$. Les négociations entre la Chambre d'agriculture, les syndicats agricoles, la DREAL et Angers Loire Métropole dans le cadre de l'élaboration du SCoT d'Angers se centrent notamment sur ces questions : « C'est du travail de fond qui n'est pas évident et qui n'est pas accepté facilement par les exploitants qui sont dans un système et qui ne voient pas pourquoi on viendrait leur parler biodiversité chez eux " (Chargé de mission trame verte et bleue à la DREAL des Pays-de-Loire). En effet, de nombreux agriculteurs rejettent la création d'un maillage dense sur leur exploitation, par peur que ces tracés ne compromettent l'efficacité et le caractère fonctionnel des structures agricoles. Bien qu'aujourd'hui aucune modification des pratiques agricoles ne soit imposée, ces agriculteurs redoutent l'évolution du cadre législatif dans un sens plus contraignant. Bref, pour justifier les trames aux yeux du monde agricole, il n'est pas possible de se contenter d'invoquer la préservation de la biodiversité. Il faut mobiliser d'autres arguments, compréhensibles à l'intérieur des régimes de justification de ce monde ${ }^{10}$. Mais justement : "La trame verte et bleue dans le SCoT, c'est un moyen supplémentaire pour défendre le maintien des terres agricoles [...] en zone périurbaine " (Chargé de mission trame verte et bleue à la DREAL des Pays-de-Loire). Et c'était déjà dans cet objectif de maintenir une agriculture périurbaine et de limiter la "consommation d'espace pour préserver les terres agricoles" qu'une réflexion spécifique sur l'avenir de l'agriculture avait été engagée dans le cadre de l'élaboration du SCOT du pays de Rennes ${ }^{11}$.

8 D'autres débats, qui relèvent du génie écologique, portent sur la possibilité de considérer les alignements d'arbres plantés en milieu urbain comme éléments de la trame. Selon le 
directeur adjoint du service des parcs et jardins d'Angers, c'est le cas ; ces alignements font bien partie de la trame :

" [La trame verte à Angers] existe de manière partielle. Ça dépend de ce que c'est qu'une trame. Ça me paraît vraiment difficile en ville d'avoir une trame continue. Il y a une trame qui m'intéresse beaucoup ce sont les arbres d'alignement. Ça c'est une trame intéressante. On peut la faire, pas partout, mais presque. Sinon, le terme de trame je le saisis pas totalement. Elle peut être discontinue aussi. [...] Avec les parcs Saint-Nicolas et le lac du Maine, on a une trame continue importante. Il y a aussi les arbres d'alignement et on a une trame discontinue de jetée de points verts. Il y a aussi une trame verte avec les jardins privés. Donc pour moi, ce n'est pas la trame, c'est les trames: il y a des trames continues, des trames discontinues et des trames spécifiques comme les arbres d'alignement ».

10 Mais pour ce chargé de mission Espaces verts au service voirie de la direction de l'espace public de Nantes Métropole, ce n'est pas nécessairement le cas :

11 «Un alignement d'arbres sans strate herbacée, en termes de corridor, je ne sais pas si ça fonctionne. [...] Oui, s'il y a un accompagnement en termes de strate herbacée ou arbustive au pied de l'alignement d'arbre. Dans ce cas je serai assez d'accord. [Mais] un alignement d'arbre sans la strate herbacée et arbustive, pour moi ça ne fonctionne pas en termes de corridor. Peut-être pour les oiseaux ça fonctionne, mais pour eux, la notion de corridor... ».

12 La connexion entre différents "espaces à caractère naturel » (Clergeau, 2007) ne peut donc répondre à elle seule au problème du déclin de la biodiversité. Il faut que soit également prise en compte la qualité écologique des habitats qui composent la trame. Toutes ces questions appellent une nouvelle expertise, un génie écologique, de nouveaux supports cartographiques (Liénard et Clergeau, 2011), dont les grandeurs sont bien de type industriel.

\section{Le monde civique}

13 L'argumentaire écologique, mobilisé dans le débat démocratique au nom de l'intérêt général et du bien commun, s'inscrit tout particulièrement dans le régime de justification civique. Pour $\mathrm{C}$. Lafaye et $\mathrm{L}$. Thévenot, c'est plus précisément «à travers les associations que les exigences démocratiques et civiques intègrent le mieux les préoccupations liées à l'environnement " (Lafaye et Thévenot, 1993: 510). De fait, notre enquête confirme l'engagement des associations naturalistes dans la mise en œuvre des trames. L'introduction des trames vertes dans le droit leur offre en effet un levier juridique inédit pour œuvrer à la protection de la biodiversité ordinaire : « On s'est longtemps centrés sur la protection du patrimoine naturel remarquable. [...] Aujourd'hui, l'enjeu est autant sur le patrimoine naturel ordinaire [...] qui existe du jardin de ville jusqu'au littoral. La biodiversité remarquable, on va continuer à s'en occuper, à la protéger et valoriser, mais on va aussi s'occuper de tout le reste $»^{12}$ (Président de l'association Bretagne Vivante).

14 On peut y voir une volonté de compromis entre le monde civique et le monde industriel autour de la reconnaissance de l'expertise associative. Mais les associations peuvent également s'engager sur le terrain de la critique au sein du monde civique ainsi que le soulignaient déjà Lafaye et Thévenot :

"la critique de la légalité des actions portant atteinte à l'environnement et le recours au contentieux, la remise en cause des enquêtes publiques, la dénonciation de la concertation comme simulacre, particulièrement vive dans le cas des grands projets d'aménagement, 
témoignent de cet engagement civique de l'action écologique » (Lafaye et Thévenot, 1993 :

510). de l'environnement, a intenté une action en justice afin d'obtenir l'annulation du plan local d'urbanisme (PLU) centre (couvrant les communes d'Angers, Avrillé, SaintBarthélemy-d'Anjou et Trélazé) approuvé par le conseil de la communauté d'agglomération d'Angers Loire Métropole en mai 2006. Elle a obtenu gain de cause, le tribunal administratif de Nantes ayant annulé ce PLU en avril $2009^{13}$. L'association dénonçait le manque de concertation de l'ensemble des partenaires, ainsi que le déclassement de certains espaces boisés classés qui allait, selon elle, à l'encontre des objectifs de développement durable pourtant affichés par Angers Loire Métropole (voir aussi Cormier et al., 2010). Le tribunal l'a d'ailleurs suivie sur ce point, l'un des considérants du jugement précisant qu' "en tant qu'ils ont fait le choix de ne pas conserver un classement en espace boisé pour des sites qui concourent de manière substantielle à la richesse $d u$ patrimoine paysager de l'agglomération, les auteurs du plan local d'urbanisme centre ont entaché leur décision d'une erreur manifeste d'appréciation », ceci alors que «le plan de développement durable affirme notamment [...] que l'espace naturel est "un élément constructif de l'aménagement, de l'image et de l'identité de l'agglomération" qui doit lui permettre "d'affirmer son statut de capitale écologique de l'Ouest" ». Mais alors que la question des espaces boisés classés était, selon la vice-présidente de l'association, l'entrée la plus facile et la plus solide, d'un point de vue juridique, pour obliger les élus à prendre en compte la place du végétal en ville, l'existence d'une trame verte et les spécificités écologiques d'un territoire, les élus d'Angers Loire Métropole ont déploré un jugement qui bloquait la construction de logements et mettait en péril un certain nombre d'entreprises du secteur du bâtiment, laissant parfois entendre - façon de réduire la grandeur civique de la décision en la faisant descendre du général au particulier - que l'ensemble du PLU avait été annulé à cause des arbres d'une seule place : "On a dû vous dire qu'on avait annuléle PLU pour la place de la Rochefoucauld. On n'arrive pas à se faire comprendre sur le fond. Heureusement que le juge n'a pas annulé un PLU entier pour une seule place!» (Vice-présidente de l'association Sauvegarde de l'Anjou). Du côté de la direction du développement des territoires d'Angers Loire Métropole, on soulignait toutefois, un an après le jugement, que « cette annulation $d u$ PLU et sa reprise peuvent être considérées comme des chances par rapport à la concertation, par rapport à cette affaire de la trame verte. De toutes façons, on y aurait été puisqu'à partir du moment où on l'élaborait dans le SCoT, derrière on a trois ans pour mettre en compatibilité les PLU avec le SCoT. [...] Là, il se trouve qu'effectivement on va faire les deux en même temps. Mais de toutes façons on l'aurait fait $»^{14}$.

Pour autant, si la trame verte offre aux associations naturalistes un cadre légal supplémentaire, elle reste très peu connue et très peu compréhensible par le grand public. En ce sens, elle ne donne pas encore lieu à de véritables débats publics, mais tend à rester l'apanage de quelques experts, ce qui pourrait donner lieu à une critique depuis le monde civique mais aussi le monde de l'opinion, tant la référence au public, cet «être ambigu, favorise les passages » entre ces deux mondes (Boltanski et Thévenot, 1991 : 386).

\section{Le monde de l'opinion}

Pour Lafaye et Thévenot, "la cause écologique tend à s'inscrire dans le mode d'évaluation du renom lorsque ses tenants cherchent à attirer l'attention de l'opinion en recourant aux médias, aux 
campagnes publicitaires ou à la mobilisation de personnalités susceptibles, par leur notoriété de donner un retentissement aux actions menées » (Lafaye et Thévenot, 1993 : 507). Or la trame verte est encore trop confidentielle pour mobiliser les médias. Très technique et portée par un débat d'experts (y compris associatifs), la trame verte urbaine n'est pas véritablement identifiée, ne constitue pas - encore? - une cause notoire qui la porterait au regard de l'opinion. Elle ne convoque pas de figure médiatique ou de leader d'opinion qui aiderait à lui donner consistance aux yeux des citadins.

Pour autant, dans une moindre mesure et au sein de cercles d'initiés (ingénieurs territoriaux, élus, etc.), elle peut participer au renom d'une métropole. Au-delà, par exemple, de la notoriété de la ville de Rennes pour son passage précoce à la gestion différenciée, l'articulation du SCOT du pays de Rennes autour de sa "trame verte et bleue ", soit "le réseau des vallées avec le fonctionnement biologique qu'il sous-tend ${ }^{15}$ ", continue à alimenter cette réputation à l'échelle nationale. En mobilisant les acteurs locaux autour du diagnostic environnemental lors de la phase prospective préliminaire à la rédaction du Plan d'aménagement et de développement durable (PADD) et du DOG (septembre 2004 à juin 2005), le SCoT du pays de Rennes a largement contribué à la renommée environnementale de la métropole. Nantes, également, se félicite non seulement d'avoir fait partie des villes pionnières en matière de gestion différenciée, mais aussi en matière de «trames» et de "corridors", d'abord sous le nom de "coulées vertes»: "On a eu la chance d'avoir des coulées vertes, une trame d'eau accompagnée d'une ambition politique forte qui a commencé depuis longtemps. [...] La largeur de la coulée verte de la Chézine et du Cens est de 100 à 120 mètres. Et quand on parle des trames vertes, c'est important » (directrice du bureau d'étude du Service des espaces verts et de l'environnement, ville de Nantes). De fait, Nantes peut s'appuyer très fortement sur son réseau hydrographique (Loire, Cens, Chézine, Erdre, Sèvre nantaise) pour définir sa trame. Mais la métropole nantaise cherche aujourd'hui à aller plus loin : restauration de la vallée du Cens entre 2003 et 2010 (avec réhabilitation des berges, mise en place d'une passe à poisson et amélioration des continuités piétonnières), inventaire en cours des zones humides, création de trois "forêts urbaines" sur une surface totale de 1400 ha, engagement vers un objectif de "zéro phyto ", protection des espèces rares ou endémiques, comme l'angélique des estuaires (Angelica heterocarpa), la fritillaire pintade (Fritillaria meleagris), le scirpe triquètre (Schoenoplectus triqueter) ou la dorine à feuilles opposées (Chrysosplenium oppositifolium $)^{16}$. Toutes ces mesures, outre qu'elles mettent en œuvre un génie écologique, ont contribué à la renommée de cette ville qui en octobre 2010 a été élue «Capitale verte de l'Europe » pour l'année 2013 par la Commission européenne.

\section{Le monde domestique}

Lafaye et Thévenot soulignaient que « tout un ensemble d'arguments centrés sur la défense de l'environnement » trouvaient dans le monde domestique " une matrice propre à les accueillir » (Lafaye et Thévenot, 1993: 505). Ils ajoutaient que «c'est souvent le recours à la notion de patrimoine qui permet d'intégrer des préoccupations ayant trait à la sauvegarde de l'environnement dans une justification fondée sur le respect des traditions" (ibid.: 506). L'intégration dans les trames vertes de certains espaces qualifiés de patrimoniaux le confirme. Dans le cadre du SCoT du pays de Rennes, par exemple, un consensus s'est constitué entre les communes au sujet de la préservation du bocage comme "terrain identitaire commun» (responsable environnement et aménagement durable, agence 
d'urbanisme). De même, l'aménagement de la ZAC de la Courrouze et la réhabilitation du quartier de Beauregard à Rennes ont beaucoup insisté, entre autres, sur la conservation d'éléments paysagers constitutifs d'un patrimoine naturel. À Beauregard, la trame bocagère a été identifiée comme l'armature autour de laquelle l'urbanisation devait prendre forme. Les arbres et les haies ont été conservés. Quant à la ZAC de la Courrouze, elle a intégré en amont l'expertise d'écologues afin de réaliser un inventaire de la biodiversité présente, définie comme le "capital vert» de l'espace (entretien avec le chargé d'opération de la ZAC à la société d'économie mixte Territoires \& Développement). On voit ainsi se dessiner un compromis entre le monde industriel, dans sa version écologique, et le monde domestique.

Pour le président de l'association Bretagne vivante, la trame répond à un objectif écologique de conservation de la biodiversité mais peut également contribuer à relier les habitants à leur territoire : "C'est quoi aujourd'hui ce qui relie un habitant à son territoire? Et bien, de moins en moins de choses. Donc peut-être que ce qui fait la caractéristique du sol, du terrain, de la flore et de la faune où on habite, ça serait déjà une manière d'habiter un peu un territoire ». La biodiversité locale contribue donc à définir une identité. Elle participe au processus de territorialisation en luttant contre la standardisation et l'homogénéisation des paysages et des milieux ${ }^{17}$. C'est ainsi que le SCoT du pays de Rennes insiste sur la nécessité de préserver cette "construction culturelle " qu'est la "trame bocagère ", élément de l'« identité paysagère du Pays ». À cela s'ajoute la préservation du savoir-faire traditionnel comme le pâturage, la fauche, la taille des arbres en «ragosse »... La trame verte inscrite dans ce régime de justification réhabilite donc la biodiversité locale comme porteuse de mémoire et support de l'identité du territoire. Encore faut-il que l'échelle considérée soit suffisamment importante: si ce n'est pas le cas, la justification de type domestique tend au contraire à être déconsidérée en tant que manifestation de l'attitude NIMBY (cas, par exemple, de la prairie des Matélouères à Rennes où des habitants se sont opposés à sa transformation partielle en zone constructible).

\section{Le monde marchand}

21 Dans le monde marchand, la nature a une valeur en tant qu'elle est désirée et se voit attribuer un prix en fonction de sa rareté. Mais la fixation d'un prix des processus écologiques et de l'équilibre des écosystèmes reste difficile: les référentiels de mesure manquent ou ne font pas l'unanimité. "L'évaluation marchande se référant directement à la cause écologique ne va pas sans poser de problème. Les êtres de la nature résistent aux efforts pour les assimiler à des biens marchands, même si le montant des amendes peut contribuer à les introduire dans un espace de prix» (Lafaye et Thévenot, 1993: 508). Les "services écosystémiques » commencent seulement à faire l'objet d'évaluations économiques : fourniture de matières premières, fertilisation des sols, épuration de l'eau, pollinisation, prévention d'un certain nombre de risques (inondations, érosion des sols, etc.) ${ }^{18}$.

Lors de nos entretiens, nous avons pu noter que le débat est très vif avec certains agriculteurs et certaines organisations agricoles qui craignent notamment que les trames ne compromettent l'activité de production. Une solution, comme on l'a vu, peut être trouvée à l'intérieur même du monde industriel en faisant en sorte que les documents d'urbanisme garantissent le maintien des terres agricoles périurbaines. Mais ces solutions passent le plus souvent par un compromis entre les justifications industrielles et marchandes. L'intégration et la protection des terres agricoles comme élément des 
trames vertes est facilitée par une évolution des pratiques agricoles permettant d'associer préservation de la biodiversité, finalités productives et finalités marchandes. La réintroduction de certaines races rustiques, par exemple, associée à la fauche, est considérée comme favorable à la préservation des milieux. Nantes Métropole affirme avoir de ce point de vue "une politique agricole assez volontariste: soutien et aide aux agriculteurs qui veulent s'implanter, défricher, etc. [...] On travaille beaucoup sur les circuits courts, l'idée étant de faire venir les habitants de l'agglomération chez l'agriculteur le plus près de chez eux» (chef du service Eaux et espaces naturels, Nantes Métropole). Alors que le répertoire marchand est difficile à mobiliser pour justifier une trame verte en milieu agricole intensif, sa mobilisation reste concevable toutes les fois où il est possible de concilier l'activité agricole avec l'objectif de préservation de la biodiversité. Quand la trame verte fonctionne comme levier pour rééquilibrer un paysage, réhabiliter le bocage ou revaloriser des pratiques de culture ou de pâturage traditionnelles, elle peut se prévaloir de conduire à une agriculture labélisée ${ }^{19}$ et déboucher sur des produits valorisés par un prix, dans un compromis associant au monde marchand les mondes industriel et domestique.

\section{Le monde inspiré}

La trame verte pourrait également s'appuyer sur le régime de justification du monde inspiré. Il faudrait pour cela, par exemple, que soit fait référence à la singularité ou à l'authenticité des paysages, qui y trouvent leur grandeur et doivent être conservés ou réaménagés pour des raisons esthétiques. C'est en effet non seulement dans l'expression d'une intimité « ineffable » avec la nature mais aussi dans un compromis entre arguments écologiques et arguments esthétiques, au nom de l'harmonie et de l'équilibre, que Lafaye et Thévenot voyaient une possibilité d'intégration de la protection de l'environnement dans le monde inspiré (Lafaye et Thévenot, 1993: 506-507). Pour O. Godard, le monde inspiré pouvait même être celui de la protection de la nature pour elle-même, en tant qu'au-delà de l'homme : « la nature inspirée s'accorde bien avec l'idée de protection de la nature, en ce qu'il s'agit de soustraire les hommes à la seule emprise de leurs intérêts terrestres, domestiques ou économiques et de symboliser une transcendance par des limites inscrites sur les êtres naturels » (Godard, 1990 : 226).

De fait, la dimension esthétique des trames vertes ou plus largement de la question de l'environnement a déjà fait l'objet de plusieurs travaux (Chon et Shafer, 2009; Blanc et Lolive, 2009a et 2009b). Ce régime de justification, toutefois, n'est guère apparu dans nos entretiens, même s'il n'en est pas complètement absent. Le président de l'association naturaliste Loire Anjou Environnement, par exemple, défend l'idée que "la meilleure méthode, [...] c'est laisser la nature reprendre ses droits. [...] La meilleure chose, quand on parle exclusivement pour l'environnement, ça serait vraiment d'inciter la population à s'installer ailleurs, à la limite, de détruire les constructions et de laisser la forêt reprendre ses droits ». Mais s'agit-il ici d'une argumentation s'inscrivant dans le monde inspiré ou plutôt d'un témoignage en faveur de cette cité verte toujours en construction? Dans un autre registre, le responsable du patrimoine et directeur-adjoint de la direction des parcs et jardins de la ville d'Angers évoque la façon dont ce service cherche à combiner des couleurs "comme une partition de musique», une réflexion horticole qui, il est vrai, n'est pas spécifique à la trame verte : 
"Ce qui est frappant à Angers, c'est qu'il y a une palette de quatre couleurs. Le bleu du ciel, adouci, très doux, le blanc un peu champagne du tuffeau, le noir bleuté des pierres de schiste et le vert. Donc un palette de quatre couleurs dominantes, froides mais très vives, donc nous, en termes de fleurissement, on essaie de s'inscrire dans ces palettes ".

\section{Conclusion}

25 Même s'il n'est pas toujours facile de ranger les arguments dans un seul monde, les critiques ou les justifications étant souvent hybrides, nous pensons avoir montré en quoi les réflexions et controverses autour des trames vertes urbaines mobilisent plusieurs des différents régimes de justification identifiés par Boltanski et Thévenot (1991). Les arguments de type industriel ont été particulièrement présents dans nos entretiens. Cela est sans doute lié aux caractéristiques de notre échantillon, composé pour une bonne part d'aménageurs dont la culture, incluant désormais le génie écologique, est en effet plutôt d'ordre industriel. Les associations, lorsqu'elles revendiquent une expertise, tendent aussi à s'inscrire dans ce monde. Il faut d'ailleurs relever que l'article L 371-1 du code de l'environnement, issu de la loi Grenelle 2, déjà cité en introduction, qui annonce que les trames vertes et bleues " ont pour objectif d'enrayer la perte de biodiversité ", ajoute aussitôt que cela passe par "la préservation", mais aussi " la gestion et la remise en bon état des milieux nécessaires aux continuités écologiques ». Mais les arguments d'ordre civique, ainsi que ceux faisant appel à l'opinion ou à la tradition (monde domestique) ne sont pas absents. Les arguments de type marchand ou inspiré, par contre, sont peu présents dans nos entretiens. La dimension esthétique, au sens étymologique des liens "sensibles » à l'environnement (Blanc et Lolive, 2009a), était plus présente dans une autre série d'entretiens auprès d'usagers d'espaces intégrés dans les trames vertes des agglomérations étudiées, certaines des personnes rencontrées tenant des propos clairement « inspirés » (Le Bot et Philip, 2010). Mais ces propos se référaient alors à une intimité avec la «nature » en général, sans référence spécifique aux trames, peu connues du public ${ }^{20}$. Qu'en est-il alors de l'autonomie ou de la consolidation du mode de justification écologique?

Comme nous le soulignions en introduction, on peut penser que l'inscription des trames vertes et bleues dans le droit et les objectifs d'aménagement du territoire en tant qu'outil de la préservation de la biodiversité va dans ce sens. En effet même si l'article L 371-1 précité évoque la "gestion» des milieux, cette gestion est justifiée par l'objectif de préserver la biodiversité. Toutefois, parmi les acteurs interrogés ou évoqués dans nos entretiens, seuls des représentants d'associations naturalistes ont vraiment insisté sur ce point. Nous avons déjà cité plus haut les propos tenus lors des ateliers thématiques dans le cadre de l'élaboration du SCoT d'Angers. Nous pourrions ajouter les regrets du président de l'association Loire Anjou environnement sur le fait que «l'écologie passe après l'économique et le social et même dans ce chapitre de protection de la biodiversité et de la trame ", alors que, selon lui, comme nous l'avons vu plus haut, " la meilleure méthode, c'est l'absence d'action, c'est ne rien faire. C'est laisser la nature reprendre ses droits ». Il n'est guère envisageable, cependant, de pouvoir partout « laisser faire la nature » (Génot, 2010). Viser l'objectif de préservation de la biodiversité y compris "ordinaire », qui peut soutenir l'hypothèse d'une consolidation de la cité verte, passe alors par des compromis avec les autres mondes. 


\section{BIBLIOGRAPHIE}

Allag-Dhuisme F., Amsallem J., Barthod C., Deshayes M., Graffin V., Lefeuvre C., Salles E. (coord), Barnetche C., Brouard-Masson J, Delaunay A., Garnier C.C., Trouvilliez J., 2010, Choix stratégiques de nature à contribuer à la préservation et à la remise en bon état des continuités écologiques - premier document en appui à la mise en œuvre de la Trame verte et bleue en France. Proposition issue du comité opérationnel Trame verte et bleue. Paris, Ministère de l'Écologie, de l'Énergie, du Développement durable et de la Mer (MEEDDM).

Berges L., Roche P. et Avon C., 2010, « Corridors écologiques et conservation de la biodiversité, intérêts et limites pour la mise en place de la Trame verte et bleue ", Sciences Eaux et Territoires, $\mathrm{n}$ - 3, p. 34-39. http://hal.archives-ouvertes.fr/hal-00545104/fr/, consulté le 10 nov. 2011.

Blanc N. et Lolive J., 2009a, « Vers une esthétique environnementale : le tournant pragmatiste », Natures Sciences Sociétés, vol. 17, n 3, p. 285-292.

Blanc N. et Lolive J., 2009b, « La restauration écologique : une nouvelle formation du monde ? ", Cybergeo : European Journal of Geography [En ligne] article 479, mis en ligne le 14 décembre 2009. URL : http://cybergeo.revues.org/22806, consulté le 27 février 2012.

Boltanski L. et Thévenot L., 1991, De la justification - Les économies de la grandeur, Paris, Gallimard.

Chon J. et Shafer C. S., 2009, « Aesthetic Responses to Urban Greeway Trail Environments », Landscape Research, vol. 34, $\mathrm{n}^{\circ}$ 1, p. 83-104.

Clergeau P., 2007, Une écologie du paysage urbain, Rennes, Apogée.

Cormier L., Bernard de Lajartre A. et Carcaud N., 2010, « La planification des trames vertes, du global au local : réalités et limites ", Cybergeo : European Journal of Geography [En ligne], article 504, mis en ligne le 06 juillet 2010. URL : http://cybergeo.revues.org/23187, consulté le 26 juin 2012.

Descola P., 2005, Par-delà nature et culture, Paris, Gallimard.

Génot J.-C., 2010, La nature malade de la gestion. La gestion de la biodiversité ou la domination de la nature, Paris, Sang de la Terre.

Godard O., 1990, « Environnement, modes de coordination et systèmes de légitimité : analyse de la catégorie de patrimoine naturel », Revue économique, vol. 41, n 2, p. 215-242.

Gramaglia C., 2008, « Des poissons aux masses d'eau : les usages militants du droit pour faire entendre la parole d'êtres qui ne parlent pas », Politix, vol. 3, n 83, p. 133-153.

Gramaglia C., 2009, « Passions et savoirs contrariés comme préalables à la constitution d'une cause environnementale. Mobilisation de pêcheurs et de juristes pour la protection d'une rivière ", Revue d'anthropologie des connaissances, vol. 3, n 3, p. 406-431.

Lafaye C. et Thévenot L., 1993, « Une justification écologique ? Conflits dans l'aménagement de la nature ", Revue française de sociologie, vol. 34, n 4, p. 495-524.

Latour B., 1991, Nous n'avons jamais été modernes, Essai d'anthropologie symétrique, Paris, La Découverte. 
Latour B., 1995, « Moderniser ou écologiser ? À la recherche de la "septième" cité », Ecologie Politique, $n^{\circ}$ 13, p. 5-27. http://www.bruno-latour.fr/articles/article/059.html, consulté le 10 nov. 2011.

Le Bot J.-M. et Philip F., 2011, « La nature des citadins », Penn ar Bed, n² 210, p. 3-40.

Lienard S. et Clergeau P., 2011, « Trame Verte et Bleue : Utilisation des cartes d'occupation du sol pour une première approche qualitative de la biodiversité ", Cybergeo : European Journal of Geography [En ligne] article 519, mis en ligne le 01 mars 2011. URL : http:// cybergeo.revues.org/23494, consulté le 10 nov. 2011.

Menozzi M.-J., 2007, « “Mauvaises herbes”, qualité de l'eau et entretien des espaces », Natures Sciences Sociétés, vol. 15, n², p. 144-153.

\section{NOTES}

1. Pour mémoire, rappelons que ces six modes de justification sont ceux de la cité marchande, de la cité inspirée, de la cité domestique, de la cité de l'opinion, de la cité civique et de la cité industrielle.

2. Loi 2009-967 du 3 août 2009 dite Grenelle 1 et loi 2010-788 du 12 juillet 2010 dite Grenelle 2. Voir aussi la contribution de Nathalie Blanc dans ce même numéro.

3. Article 121 de la loi Grenelle 2 créant l'article L371-1 du Code de l'environnement relatif à la trame verte et bleue.

4. Ibid., alinéas 1 et 2 .

5. "Évaluation des Trame vertes urbaines et élaboration de référentiels: une infrastructure entre esthétique et écologie pour une nouvelle urbanité » (2008-2013), programme de recherche financé par l'Agence Nationale de la Recherche (ANR) dans le cadre de son appel à projet Villes Durables - http://www.trameverteurbaine.com/

6. Syndicat Mixte de la région angevine (SMRA) et Sémaphores, Synthèse des ateliers avec les associations et les professionnels (février-mars 2010). En ligne : http://www.scot-regionangevine.fr/ syntheseconcertation2.pdf, consulté le 15 avril 2011. Au niveau national, la version consolidée par l'État (juillet 2010) du premier guide issu des propositions du Comité Opérationnel Trame verte et bleue (ultérieurement COMOP) indique, de son côté, que "la mise en œuvre d'une Trame verte et bleue permet aussi de répondre à cette demande [sociale d'espace de nature] mais doit satisfaire en priorité les objectifs de conservation de la biodiversité (maîtrise de la fréquentation, sensibilisation aux enjeux et objectifs poursuivis) " (Allag-Dhuisme et al., $2010: 29$ ).

7. Un participant à la journée technique Plante \& Cité de Rennes, le 22 septembre 2011, soulignait le caractère trompeur de cette expression : comme s'il s'agissait de soigner les plantes, quand il s'agit en réalité de les tuer. Mais d'un autre côté, un article publié dans la revue Horticulture et Paysage (juin 2010) et signé par l'ancien directeur du Service des espaces verts et de l'environnement de Nantes appelait à «sauver le vrai jardinier » menacé par les nouvelles « pratiques philosophico-écologiques ».

8. Les propositions du COMOP s'inscrivent dans une « approche d'ensemble de la biodiversité, appuyée par la prise en compte des acquis scientifiques récents en matière d'habitats naturels et de structures paysagères, [qui] a conduit à soutenir une nouvelle dynamique de protection et de restauration qui ne soit pas exclusivement centrée sur une biodiversité remarquable. Les interactions au sein d'un écosystème concernent toutes les espèces végétales et animales et pas seulement les plus rares et les plus menacées. Ce faisant, un intérêt nouveau s'est fait jour pour une biodiversité parfois qualifiée "d'ordinaire" qui rend aussi des services, divers et multiples souvent irremplaçables pour l'homme et recouvrant d'ailleurs des espèces communes en voie de régression, telles les abeilles » (op. cit. : 6) 
9. Le COMOP note à ce sujet que : "Les mutations des pratiques agricoles ont entraîné, notamment en zone de plaine, la remise en cause de certaines structures paysagères au détriment des espèces qui y sont inféodées et de la qualité et de la diversité des paysages (banalisation, homogénéisation). Pourtant, il existe des synergies entre agriculture, biodiversité et paysage. Ainsi, la préservation et la remise en bon état des continuités écologiques par la Trame verte et bleue aura pour conséquence de contribuer à la protection, à la gestion et à l'aménagement de ces paysages, aussi bien pour l'homme que pour la nature " (op. cit. : 27-28).

10. Pour éviter tout malentendu, précisons que ces deux dernières occurrences du terme «monde » doivent être entendues au sens banal de « groupe social » et non au sens de Boltanski et Thévenot.

11. Sollicité par le syndicat mixte du SCOT, le Conseil de développement économique et social du pays et de l'agglomération de Rennes (CODESPAR) a mis en place un groupe de travail « Avenir de l'agriculture » qui s'est réuni entre septembre et novembre 2006. Cf. « Agriculture(s) périurbaine (s). Quelles perspectives dans le pays de Rennes?", Les contributions du Codespar, décembre 2006, ainsi que la notice du SCoT du pays de Rennes, p. 22.

12. L'association Bretagne Vivante gère plus d'une centaine d'espaces protégés dont 5 réserves naturelles nationales.

13. L'association Sauvegarde de l'Anjou est une fédération départementale d'associations issue de l'association Sauvegarde du Vieil Angers créée en 1965. Elle a obtenu l'agrément des pouvoirs publics en 1978 au titre de l'environnement, de l'aménagement du territoire et de l'urbanisme. Son évolution et son mode d'action présentent des points communs avec ceux de l'association ANPER-TOS (Gramaglia, 2008 ; Gramaglia, 2009). Sa vice-présidente, par exemple, est une juriste spécialisée en droit de l'environnement.

14. Le SCoT Pays-de-Loire Angers a depuis été approuvé, en novembre 2011, par le comité syndical du Syndicat mixte de la région angevine (SMRA).

15. SCoT du pays de Rennes, Rapport de présentation, décembre 2007, p. 66.

16. La première est protégée au niveau européen (directive Habitats) et figure en France dans le livre rouge des espèces menacées. La seconde fait l'objet d'arrêtés de protection dans plusieurs départements français dont la Loire-Atlantique. Le troisième fait l'objet d'un arrêté de protection dans plusieurs régions dont la région Pays-de-Loire. La quatrième est protégée en France en région Centre.

17. Le COMOP note à ce sujet que "la mosaïque de milieux induite par la Trame verte et bleue (les différents habitats naturels, les haies, fossés, bordures de cours d'eau, prairies, petites friches, talus et bosquets, etc.) donne généralement du caractère au paysage: ils font reculer sa banalisation et son uniformisation. Cette mosaïque structure le paysage en améliorant la perception visuelle des espaces seminaturels et en permettant la conservation d'une ambiance agréable dans les zones aménagées, satisfaisant ainsi les aspirations des populations vis-à-vis de leur cadre de vie » (op. cit. : 29).

18. Le COMOP note à ce sujet que « la Trame verte et bleue a pour ambition de préserver la biodiversité en maintenant ou en restaurant le maillage et la fonctionnalité des écosystèmes. L'amélioration de la fonctionnalité des écosystèmes et la préservation des espaces de la Trame verte et bleue (réservoirs de biodiversité, corridors, cours d'eau) se traduiront par une amélioration des "services rendus" par ces écosystèmes à la collectivité, c'est-à-dire des bénéfices que les humains peuvent tirer des écosystèmes. Ils concernent des domaines très variés, dans certains domaines de la production, ou, par exemple, la lutte contre les inondations, l'épuration de l'eau et la pollinisation des plantes cultivées. Ces services deviennent peu à peu reconnus, en particulier certains d'entre eux font l'objet d'évaluations économiques " (op. cit. : 28).

19. C'est ce qu'affirme le COMOP : «bien des éléments paysagers, parties prenantes de la Trame verte et bleue, participent à caractériser des territoires régionaux et contribuent à l'image des activités agricoles en étant le support à la relation "qualité des paysages - qualité des produits" importante tout particulièrement pour les productions labellisées. On peut imaginer une valorisation de produits mettant en avant la 
préservation de la biodiversité tout comme certains produits se réclament par exemple d'une appartenance au territoire d'un parc naturel régional ou participant à la protection d'une espèce » (op. cit. : 31).

20. Il n'est pas certain en outre, mais cela demanderait une autre discussion, que l'approche « esthétique » ou « sensible » corresponde au seul monde inspiré.

\section{RÉSUMÉS}

En 1993, Lafaye et Thévenot se demandaient s'il n'était pas possible de définir un septième mode de justification, celui de la cité verte, aux côtés des six autres définis par Boltanski et Thévenot (1991). En s'appuyant sur des entretiens réalisés auprès d'acteurs institutionnels intervenant dans les débats autour des trames vertes urbaines de trois métropoles de l'ouest de la France (Angers, Nantes et Rennes), l'article montre que, près de vingt ans plus tard, la justification de ces trames fait largement appel au six cadres argumentatifs préexistants. Bien que la loi Grenelle 2 définissant les trames vertes et bleues insiste sur l'objectif de préserver la biodiversité pour elle-même, les promoteurs locaux de ces trames procèdent à des traductions qui aboutissent à des compromis entre mondes.

Urban green frames, a new basis for a green city? In 1993, Lafaye and Thevenot put forward the hypothesis of a seventh "order of worth" - which has been called the "green city" - besides the six other "orders of worth" identified by Boltanski and Thévenot. Based on semi-directive interviews with public officers and members of non-governmental organizations engaged in the debates about urban green frames of three metropolitan areas in the West of France (Angers, Nantes and Rennes), the current paper shows that, after nearly 20 years, urban green frames take their justification principles from the six pre-existing "orders of worth". Although "Grenelle 2" French regulation, which defines the green and blue frames, emphasizes the necessity to protect biodiversity for itself, green frames local developers are willing to translate this necessity and achieving compromises between worlds.

\section{INDEX}

Keywords : urban green frames, orders of worth, principles of justification, sociology, France

Mots-clés : trames vertes urbaines, principes de justification, légitimité, sociologie, France

\section{AUTEURS}

\section{JEAN-MICHEL LE BOT}

Jean-Michel Le Bot est Maître de conférences de sociologie, Centre interdisciplinaire d'analyse des processus humains et sociaux - (CIAPHS), Université Rennes 2, place du recteur Henri Le Moal, CS 24307, 35043 Rennes. jean-michel.lebot@univ-rennes2.fr 


\section{FRANÇOISE PHILIP}

Françoise Philip est Post-doctorante en sociologie, Centre interdisciplinaire d'analyse des processus humains et sociaux - (CIAPHS), Université Rennes 2, place du recteur Henri Le Moal, CS 24307, 35043 Rennes. philip_francoise@yahoo.fr 